\title{
Differential engagement of ORAI1 and TRPC1 in the induction of vimentin expression by different stimuli
}

\author{
Teneale A. Stewart ${ }^{1,2,3}$. Iman Azimi ${ }^{1,2,3,4}$. Daneth Marcial ${ }^{1}$ - Amelia A. Peters ${ }^{1}$ - Silke B. Chalmers ${ }^{1}$. \\ Kunsala T. D. S Yapa ${ }^{1}$ - Erik W. Thompson $\mathbb{D}^{3,5,6} \cdot$ Sarah J. Roberts-Thomson ${ }^{1}$ • Gregory R. Monteith ${ }^{1,2,3}$
}

Received: 13 February 2019 / Revised: 25 April 2019 / Accepted: 9 May 2019 / Published online: 26 June 2019

(c) The Author(s), under exclusive licence to United States and Canadian Academy of Pathology 2019

\begin{abstract}
The $\mathrm{Ca}^{2+}$ signal is essential in both hypoxia- and epidermal growth factor (EGF)-mediated epithelial to mesenchymal transition (EMT) in MDA-MB-468 breast cancer cells. This finding suggests that $\mathrm{Ca}^{2+}$-permeable ion channels participate in the induction of expression of some mesenchymal markers such as vimentin. However, the ion channels involved in vimentin expression induction have not been fully characterized. This work sought to define how differential modulation of the calcium signal effects the induction of vimentin and the $\mathrm{Ca}^{2+}$ influx pathways involved. We identified that the intracellular $\mathrm{Ca}^{2+}$ chelator EGTA-AM, cytochalasin D (a modulator of cytoskeletal dynamics and cell morphology), and the sarco/endoplasmic reticulum ATPase inhibitor thapsigargin are all inducers of vimentin in MDA-MB-468 breast cancer cells. EGTA-AM- and thapsigargin-mediated induction of vimentin expression in MDA-MB-468 cells involves storeoperated $\mathrm{Ca}^{2+}$ entry, as evidenced by sensitivity to silencing of the molecular components of this pathway, STIM1 and ORAI1. In stark contrast, cytochalasin D-mediated vimentin induction was insensitive to silencing of ORAI1, despite sensitivity to silencing of its canonical activator the endoplasmic reticulum $\mathrm{Ca}^{2+}$ sensor STIM1. Cytochalasin D-mediated vimentin induction was, however, sensitive to silencing of another reported STIM1 target, TRPC1. Subsequent studies identified that EGTA-AM-induced vimentin expression also partially involved a TRPC1-dependent pathway. These studies define a complex interplay between vimentin expression in this model and the specific $\mathrm{Ca}^{2+}$-permeable ion channels involved. The complexity in the engagement of different $\mathrm{Ca}^{2+}$ influx pathways that regulate vimentin induction are opportunities but also potential challenges in targeting $\mathrm{Ca}^{2+}$ signaling to block EMT in cancer cells. Our findings further highlight the need to identify potential indispensable ion channels that can regulate induction of specific mesenchymal markers via different stimuli.
\end{abstract}

\section{Introduction}

The plasticity of cancer cells allows the acquisition of features that can contribute to disease progression [1]. In breast

Supplementary information The online version of this article (https:// doi.org/10.1038/s41374-019-0280-3) contains supplementary material, which is available to authorized users.

Gregory R. Monteith

gregm@uq.edu.au

1 School of Pharmacy, The University of Queensland, Brisbane, QLD, Australia

2 Mater Research Institute, The University of Queensland, Brisbane, QLD, Australia

3 Translational Research Institute, Brisbane, QLD, Australia cancer, the conversion of epithelial cells to a more invasive and therapy-resistant phenotype can be the consequence of epithelial to mesenchymal transition (EMT) [2, 3]. Conditions within the tumor microenvironment, such as specific growth factors and hypoxia, are often inducers of EMT $[1,4,5]$. The conversion of some breast cancer cells to a more mesenchymal phenotype is associated with the loss of epithelial markers such as E-cadherin and claudin $4[1,6]$. EMT is also associated with acquisition of mesenchymal

4 Division of Pharmacy, College of Health and Medicine, University of Tasmania, Hobart, TAS, Australia

5 Institute of Health and Biomedical Innovation and School of Biomedical Sciences, Queensland University of Technology, Kelvin Grove, QLD, Australia

6 The University of Melbourne Department of Surgery, St Vincent's Hospital, Melbourne, VIC, Australia 
markers such as the transcription factors Twist, Zeb, and Snail, and is linked to stemness via an increase in CD44 and/or a downregulation of CD24 cell adhesion molecule levels $[7,8]$. In many models of breast cancer cells, EMT also results in a pronounced upregulation of the intermediate filament protein vimentin, which is a regulator of cellular motility, adhesion, and signaling [9]. The identification of regulators of EMT in breast cancer cells represents a new suite of drug targets for therapeutic intervention of metastatic progression and/or drug resistance [3, 10, 11].

Recently, studies have identified the remodeling of $\mathrm{Ca}^{2+}$ signaling and/or changes in the expression of components of $\mathrm{Ca}^{2+}$ transport as a consequence of EMT in cancer cells $\left([5,12-14]\right.$ and reviewed in [15]). The $\mathrm{Ca}^{2+}$ signal itself is also a key regulator of the induction of EMT by some stimuli. For example, EMT induced by both epidermal growth factor (EGF) and hypoxia is inhibited by the suppression of $\mathrm{Ca}^{2+}$ signals by intracellular $\mathrm{Ca}^{2+}$ buffering using BAPTAAM in MDA-MB-468 breast cancer cells [5]. Similarly, BAPTA-AM reduces EMT induced by acidity in PANC-1 and BxPC-3 pancreatic cancer cells [16] and by transforming growth factor- $\beta 1$ in A549 lung cancer cells [17]. These studies collectively place the $\mathrm{Ca}^{2+}$ signal as a vital cellular signal in the induction and/or maintenance of EMT. However, the contribution of specific $\mathrm{Ca}^{2+}$ influx pathways to EMT induction is likely to be dependent on the broader cellular context, that is, the nature of the inducer and/or the type and features of the cancer cell line under investigation. For example, the plasma membrane localized store-operated $\mathrm{Ca}^{2+}$ entry channel component ORAI1 is not a regulator of vimentin expression induced by EGF in MDA-MB-468 breast cancer cells [5], but is a regulator of vimentin expression induced by fibroblast growth factor isoform 4 in A549 and H1299 lung cancer cells [18]. A better understanding of the landscape of EMT induction, through the identification of the molecular players involved in $\mathrm{Ca}^{2+}$ signal dependent induction of vimentin, a classic mesenchymal marker, will provide new insights into this important process.

The differential contribution of $\mathrm{Ca}^{2+}$ signaling to EMT induction with different stimuli is likely to be complex. Diversity of $\mathrm{Ca}^{2+}$ influx mechanisms is reflected in studies assessing store-operated $\mathrm{Ca}^{2+}$ entry. The canonical pathway for the refilling of $\mathrm{Ca}^{2+}$ stores first involves the detection of $\mathrm{Ca}^{2+}$ store depletion by the endoplasmic reticulum $\mathrm{Ca}^{2+}$ sensor STIM1, followed by activation of $\mathrm{Ca}^{2+}$ influx via STIM1 interaction with ORAI1 hexameric $\mathrm{Ca}^{2+}$ channels $[19,20]$. However, in some cellular systems and/or stimuli, STIM1 can activate $\mathrm{Ca}^{2+}$ influx that is TRPC1-dependent, with possible differential contribution of ORAI1 [21-24]. Context dependent contributions of TRPC 1 are reflected in the ability of TRPC1 silencing to attenuate phosphorylation of the transcription factor STAT3 induced by hypoxia but not EGF in MDA-MB-468 breast cancer cells [25]. Despite the aforementioned studies, the potential contribution of STIM1, ORAI1, and TRPC1 in the regulation of vimentin induction associated with different mediators of EMT has not been fully explored.

Buffering of increases in cytosolic $\mathrm{Ca}^{2+}\left(\left[\mathrm{Ca}^{2+}\right]_{\mathrm{CYT}}\right)$ with the fast $\mathrm{Ca}^{2+}$ buffer BAPTA-AM can attenuate both EGFand hypoxia-induced vimentin expression in MDA-MB-468 breast cancer cells [5]. However, these same studies observed a modest increase in basal vimentin expression by prolonged incubation with the slower intracellular $\mathrm{Ca}^{2+}$ buffer EGTA-AM. Intracellular EGTA is unable to buffer the highly localized increases in free $\mathrm{Ca}^{2+}$ that may occur near the mouth of $\mathrm{Ca}^{2+}$ channels upon their opening [26]. Numerous studies have reported the differential effects of BAPTA-AM and EGTA-AM on cellular events such as gene transcription [27-30]. However, the possible effect of EGTA-AM on vimentin expression has not been fully evaluated nor has the contribution of the $\mathrm{Ca}^{2+}$ signal or the molecular components involved in this type of EMT induction.

In this study, the ability of EGTA-AM to induce EMT was assessed in MDA-MB-468 breast cancer cells. The molecular pathways involved in EGTA-AM-mediated induction of the mesenchymal EMT marker vimentin involved components that do not contribute to other inducers of EMT, including the inhibitor of actin polymerization, cytochalasin D. Our results also provide another example of the differential contribution of STIM1 to events regulated by ORAI1 and TRPC1, and identify that the STIM1 contribution is dependent on the context of the type of cellular stimuli in MDA-MB-468 breast cancer cells.

\section{Materials and methods}

\section{Cell culture and reagents}

MDA-MB-468 breast cancer cells were maintained in DMEM with high glucose (D6546; Sigma-Aldrich, St Louis, MO, USA), supplemented with $10 \%$ fetal bovine serum (FBS, GE Healthcare) and 4mM L-glutamine (25030; Life Technologies, Carlsbad, CA, USA). Cells were incubated at $37{ }^{\circ} \mathrm{C}$ with $5 \% \mathrm{CO}_{2}$ in a humidified atmosphere. For studies assessing the effect of intracellular $\mathrm{Ca}^{2+}$ chelation, cytochalasin D (250255; Calbiochem) or thapsigargin (T9033; Sigma-Aldrich) on EMT marker expression, $2 \times 10^{4}$ cells were plated in 96-well tissue culture plates. After leaving cells to attach overnight, complete medium was replaced with serum-reduced medium (SRM, 0.5\% FBS) for $24 \mathrm{~h}$. For EGTA-AM studies, cells were incubated for $1 \mathrm{~h}$ with $100 \mu \mathrm{M}$ EGTA-AM (E1219; Invitrogen, Carlsbad, CA, USA) or DMSO control $(0.2 \%$ v/v, Sigma- 
Aldrich), before medium was replaced with fresh SRM for the remainder of the experiment. Cytochalasin D and thapsigargin were used at a final concentration of $300 \mathrm{nM}$ and $100 \mathrm{nM}$, respectively, or DMSO control $(0.1 \%)$ and cells were treated for $24 \mathrm{~h}$ prior to protein and/or RNA isolation. For inhibitor studies, MDA-MB-468 cells were cultured as described above, but were pretreated for $1 \mathrm{~h}$ with Y-27632 (Y0503; Sigma-Aldrich) or YM58483 (Y4895; Sigma-Aldrich) at the concentrations indicated. Inhibitor treatment was maintained for $24 \mathrm{~h}$ prior to protein and/or RNA isolation. MDA-MB-468 cell line authenticity was confirmed via STR profiling using the GenePrint 10 System (Promega, Madison, WI, USA) by the Queensland Institute of Medical Research Berghofer Medical Research Institute and cells routinely tested negative for mycoplasma (Lonza, Basel, Switzerland).

\section{Immunoblotting}

Total cellular protein was extracted using protein lysis buffer supplemented with protease and phosphatase inhibitors (Roche Applied Science, Penzburg, Germany) and immunoblotting was performed using a vimentin antibody (V6389; Sigma-Aldrich) at a dilution of 1:750. PVDF membranes were incubated with vimentin antibody overnight at $4{ }^{\circ} \mathrm{C}$ or for $1 \mathrm{~h}$ at room temperature with $\beta$-actin (A5441; Sigma-Aldrich) at 1:10,000, as previously described [5]. Protein quantification was performed using the Quantity One Software "rolling-ball" method (Bio-Rad, Hercules, CA, USA) and protein band intensity normalized to the loading control ( $\beta$-actin).

\section{Fluorescence microscopy}

For F-actin immunostaining, MDA-MB-468 cells were plated at a density of $1.5 \times 10^{4}$ cells/well in 96-well blackwalled imaging plates (BD Biosciences, Franklin Lake, NJ, USA). After allowing cells to attach overnight, complete medium was replaced with SRM for $24 \mathrm{~h}$. Cells were then treated with either EGTA-AM or cytochalasin D as described above for $6 \mathrm{~h}$. Cells were washed in PBS containing $\mathrm{Ca}^{2+}$ and $\mathrm{Mg}^{2+}$ and fixed in PBS containing $4 \%$ paraformaldehyde, followed by permeabilization with PBS containing $0.1 \%$ Triton X-100 (Sigma-Aldrich). Nonspecific sites were blocked with blocking buffer for $1 \mathrm{~h}$ at room temperature in a humidified chamber. For F-actin staining, cells were incubated (20 min) with Alexa-Fluor 488-Phalloidin (A12379; Life Technologies) diluted 1:40 in PBS from the methanolic stock solution. Nuclei were stained with DAPI (D1306; Life Technologies) for $10 \mathrm{~min}$ at room temperature, according to the manufacturer's directions. Images were acquired with a
Nikon Eclipse T $i$ epifluorescence microscope using a $20 \times$ objective.

\section{siRNA transfection}

For experiments assessing the effect of siRNA-mediated silencing of gene targets on EGTA-AM, cytochalasin D or thapsigargin-induced vimentin protein, MDA-MB-468 cells were plated at a density of $6 \times 10^{3}$ cells/well of a 96-well plate. After allowing cells to attach overnight, cells were cultured in the presence of siRNA-containing medium for $48 \mathrm{~h}$, followed by $24 \mathrm{~h}$ in SRM. Cells were then cultured in EGTA-AM, cytochalasin D, or thapsigargin as described above. DharmaFECT4 Transfection Reagent $(0.1 \mu \mathrm{L} /$ well) (T2004; Dharmacon, Lafayette, CO, USA) was used to deliver siRNA particles according to the manufacturer's protocol. The following Dharmacon ON-TARGETplus SMARTpool (consisting of four rationally designed siRNAs) siRNAs were used: Nontargeting (D-001810-10-05), ORAI1 (L-014998-00-0005), STIM1 (L-011785-00-0005), and TRPC1 (L-004191-00-0005). Silencing efficiency was assessed $48-72 \mathrm{~h}$ post siRNA transfection using real time RT-PCR.

\section{Real time RT-PCR}

RNA isolation, purification, and preparation of cDNA were performed as previously described [5]. The following TaqMan ${ }^{\mathrm{TM}}$ Gene Expression Assays (Applied Biosystems, Foster City, CA, USA) were used: CD24 (Hs02379687_s1), CD44 (Hs01075861_m1), CDH2 (N-cadherin; Hs00983062_m1), ORAI1 (Hs00385627_m1), SNAI1 (Snail; Hs00195591_m1), STIM1 (Hs00162394_m1), Twist1 (Hs00361186_m1), and vimentin (Hs00185584_m1). Real time RT-PCR reactions were run using a StepOnePlus Real Time PCR System (Applied Biosystems) under universal cycling conditions. Eukaryotic 18S rRNA (4319413E; Applied Biosystems) served as a reference to enable relative quantification of target gene expression. Gene expression was analyzed using the comparative $\mathrm{C}_{\mathrm{T}}$ method as described previously [31].

\section{Measurement of intracellular $\mathrm{Ca}^{2+}$}

Measurement of thapsigargin-mediated changes in storeoperated $\mathrm{Ca}^{2+}$ entry was performed by loading cells with $2 \mu \mathrm{M}$ of the cell permeant intracellular calcium indicator Fluo4, AM (F14201; ThermoFisher Scientific, Waltham, MA, USA) and imaging with a fluorometric imaging plate reader (FLIPR $^{\text {TETRA; }}$ Molecular Devices, San Jose, CA, USA) and the BD PBX no-wash $\mathrm{Ca}^{2+}$ assay kit (640175; BD Biosciences, Franklin Lakes, NJ, USA) as previously described [5], with the following change: thapsigargin (100 nM) was used to deplete endoplasmic reticulum calcium stores. Cells 
were treated with YM58483 for 15 min at room temperature prior to performing the $\mathrm{Ca}^{2+}$ assay and the inhibitor concentration was maintained throughout the experiment. Store-operated calcium entry was assessed via the peak 2/peak 1 ratio, peak 1 refers to peak $\left[\mathrm{Ca}^{2+}\right]_{\mathrm{CYT}}$ induced by the endoplasmic reticulum calcium pump inhibitor and peak 2 refers to peak $\left[\mathrm{Ca}^{2+}\right]_{\mathrm{CYT}}$ during store-operated calcium entry after readdition of extracellular $\mathrm{Ca}^{2+}$.

\section{Statistical analysis}

Statistical analysis was performed using GraphPad Prism (v6.05 for Windows). Details of statistical analyses are provided in the corresponding figure legends.

\section{Results}

Our first experiments were focused on further assessing the phenomenon whereby EGTA-AM may induce basal vimentin expression in MDA-MB-468 breast cancer cells. During this assessment, we found that EGTA-AM incubation consistently produced pronounced vimentin protein expression (Fig. 1a) and mRNA induction of vimentin and other mesenchymal markers (N-cadherin, Twist, Snail and CD44/CD24 mRNA levels) (Fig. 1b) in MDA-MB-468 breast cancer cells. EGTA-AM also produced a change in the morphology of MDA-MB-468 cells from an epithelial, cobblestone appearance, to a more spindle-like morphology with cortical actin changing to actin stress fibers (Fig. 1c). Given this change in cell morphology, we explored the possible role of cell shape changes in the induction of EMT markers in this model by assessing the effects of the actin polymerization inhibitor cytochalasin D. Similar to EGTAAM, cytochalasin D-induced vimentin expression and the induction of EMT mRNA markers in MDA-MB-468 breast cancer cells (Fig. 1d, e). Low concentration cytochalasin D $(300 \mathrm{nM})$ also induced a spindle morphology, although it produced a more localized F-actin distribution as indicated by phalloidin staining (Fig. 1f). These results demonstrate that EMT markers in MDA-MB-468 cells can clearly be induced by physical cell shape changes (via cytoskeletal reorganization) as well as chemical signals. This study therefore sought to define the cellular signaling pathways responsible for these two distinct modes of EMT induction in the MDA-MB-468 breast cancer cell line model, with a specific focus on the mechanisms associated with induction of vimentin protein expression.

Given that an important role of Rho kinase/ROCK signaling is regulation of the actin cytoskeleton [32, 33], we assessed the effect of the ROCK inhibitor Y-27632 on cytochalasin D treatment. Y-27632 was a potent inhibitor of cytochalasin D-induced vimentin expression in MDA-MB-
468 breast cancer cells (Fig. 2a). However, vimentin expression induced by EGTA-AM was insensitive to Y-27632-mediated ROCK inhibition (Fig. 2b) indicating that EGTA-AM and cytochalasin D engage at least some distinct cellular signaling pathways that lead to increased vimentin expression.

While both BAPTA-AM and EGTA-AM reduce global $\left[\mathrm{Ca}^{2+}\right]_{\mathrm{CYT}}$ levels, due to its slower rate of calcium binding, EGTA-AM will not buffer $\mathrm{Ca}^{2+}$ near the mouth of $\mathrm{Ca}^{2+}$ channels [26]. Therefore, the ability of EGTA-AM to induce vimentin expression could be due to the presence of regions of localized high $\mathrm{Ca}^{2+}$ levels at the plasma membrane following opening of $\mathrm{Ca}^{2+}$ permeable plasmalemmal ion channels in response to reduced global $\left[\mathrm{Ca}^{2+}\right]_{\mathrm{CYT}}$. Increased $\mathrm{Ca}^{2+}$ influx could be driven by reduced endoplasmic reticulum $\mathrm{Ca}^{2+}$ levels in $\left[\mathrm{Ca}^{2+}\right]_{\mathrm{CYT}}$ EGTA-AM buffered MDA-MB-468 cells. To assess this potential mechanism, components of the store-operated $\mathrm{Ca}^{2+}$ entry pathway (the pathway responsible for endoplasmic $\mathrm{Ca}^{2+}$ store refilling in epithelial cells) were silenced, specifically the $\mathrm{Ca}^{2+}$ channel component ORAI1 and the endoplasmic reticulum $\mathrm{Ca}^{2+}$ store depletion sensor STIM1 (Fig. 3a, b and supplementary Fig. 1A-D). ORAI1 and STIM1 silencing each in-part reduced EGTA-AM-induced vimentin expression (Fig. 3c), suggesting that store-operated $\mathrm{Ca}^{2+}$ entry was a partial driver for vimentin induction under these conditions. Indeed, direct promotion of store-operated $\mathrm{Ca}^{2+}$ entry via the sarcoplasmic/endoplasmic reticulum $\mathrm{Ca}^{2+}$ ATPase inhibitor thapsigargin produced a pronounced increase in vimentin expression, which was abolished by silencing of the store-operated $\mathrm{Ca}^{2+}$ entry components ORAI1 and STIM1 (Fig. 3d). Supporting the involvement of a nonidentical mechanism for cytochalasin D-induced vimentin expression in this model, cytochalasin D-induced vimentin expression was insensitive to ORAI1 silencing (Fig. 3e). However, a $\mathrm{Ca}^{2+}$ store depletion mechanism seemed to also be involved, given that silencing of STIM1 significantly reduced vimentin induction by cytochalasin D (Fig. 3e). We therefore sought to define what STIM1 activated $\mathrm{Ca}^{2+}$ influx pathway may be involved in cytochalasin D-induced vimentin expression in MDA-MB468 breast cancer cells.

Given the sensitivity of cytochalasin D-induced vimentin expression to STIM1 but not ORAI1 silencing, and reports of STIM1 activation of TRPC1 in other cell types [22-24], we assessed the effects of TRPC1 siRNA in this model. TRPC1 silencing (Fig. 4a and supplementary Fig. 1A-D) significantly reduced cytochalasin D-induced vimentin expression in MDA-MB-468 breast cancer cells (Fig. 4b), suggesting a STIM1-TRPC1 pathway that is independent of ORAI1. Like ORAI1 silencing, TRPC1 silencing also partially inhibited EGTA-AM-induced vimentin expression (Fig. 4c), suggesting EGTA-AM activates both TRPC1 
Fig. 1 EGTA-AM and cytochalasin D treatment induce features of an EMT in MDAMB-468 breast cancer cells. MDA-MB-468 cells treated with a EGTA-AM $(100 \mu \mathrm{M}, 1 \mathrm{~h})$ or d cytochalasin D (cyto D, $300 \mathrm{nM}$, $24 \mathrm{~h}$ ) have elevated protein expression of the classic EMT marker vimentin at $24 \mathrm{~h}$.

Representative immunoblot and densitometric analysis (normalized to $\beta$-actin). Relative mRNA levels of EMT-

associated genes (vimentin, Ncadherin, CD44/CD24, Twist, and Snail) following treatment of MDA-MB-468 cells with either b EGTA-AM $(100 \mu \mathrm{M}, 1$ h) or e cyto $D(300 \mathrm{nM}, 24 \mathrm{~h})$ at $24 \mathrm{~h}$. Bar graphs represent the mean \pm SD for three independent experiments. $P<0.05$ (unpaired $t$-test). Representative morphological changes in sub confluent MDA-MB-468 cells in response to treatment with either c EGTA-AM $(100 \mu \mathrm{M}, 1 \mathrm{~h})$ or $\mathbf{f}$ cyto $\mathrm{D}(300 \mathrm{nM}, 6 \mathrm{~h})$ at $6 \mathrm{~h}$.

DAPI nuclear stain (blue) and Factin (green). Scale bar $=50 \mu \mathrm{m}$
A
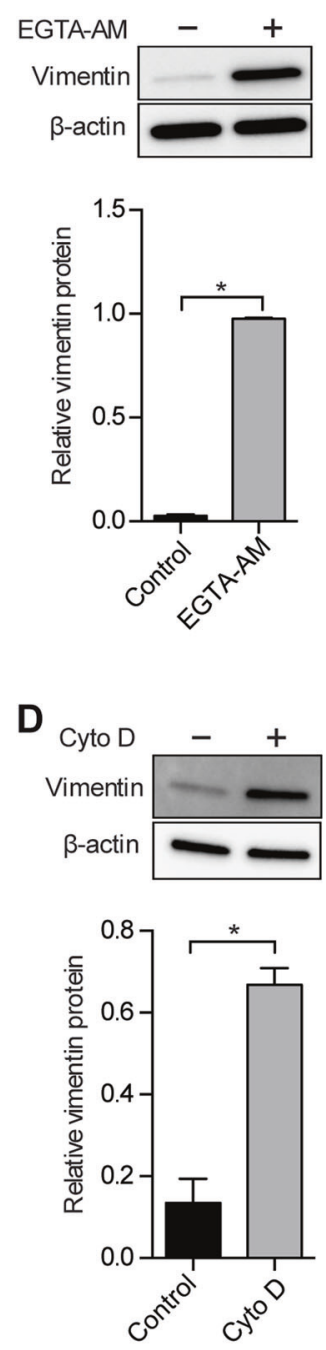

B

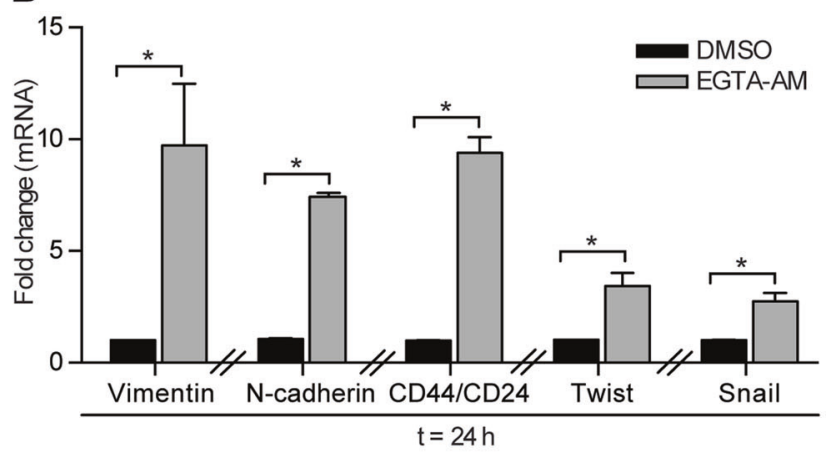

C
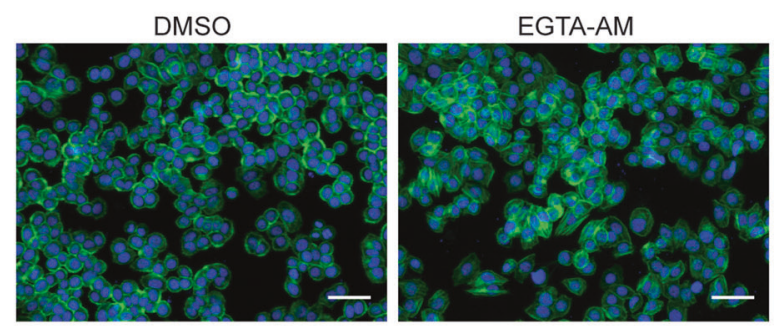

E

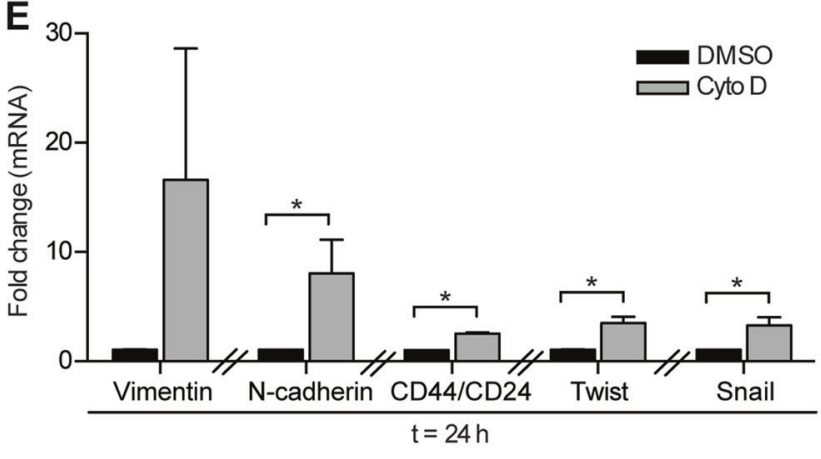

F

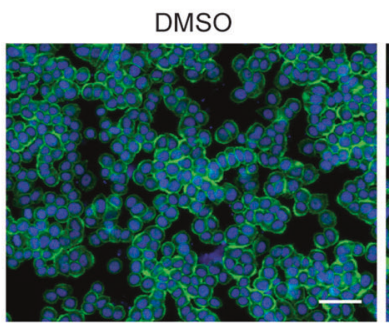

Cyto D

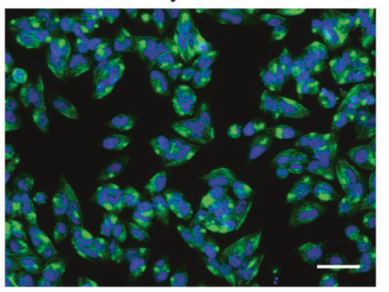

and ORAI1 mechanisms of vimentin induction. Consistent with the promotion of store-operated $\mathrm{Ca}^{2+}$ entry by TRPC1 silencing in MDA-MB-468 breast cancer cells [34], TRPC1 silencing enhanced the ability of thapsigargin to promote vimentin expression in this model (Fig. 4d). These results support differential mechanisms of $\mathrm{Ca}^{2+}$ entry to promote vimentin expression by different stimuli in MDAMB-468 cells.

The availability of pharmacological inhibitors of ORAI1mediated store-operated $\mathrm{Ca}^{2+}$ entry allowed us to further assess this pathway in the context of thapsigargin-induced vimentin expression. We first assessed the store-operated $\mathrm{Ca}^{2+}$ entry inhibitor YM58483 [35, 36]. In MDA-MB-468 breast cancer cells, YM58483 inhibited store-operated $\mathrm{Ca}^{2+}$ entry induced by thapsigargin with an IC50 of $0.35 \mu \mathrm{M}$ and with maximal inhibition achieved at $3 \mu \mathrm{M}$ (Fig. 5a, b). As expected, based on the effects of ORAI1 siRNA, pharmacological inhibition of store-operated $\mathrm{Ca}^{2+}$ entry with YM58483 almost completely abolished thapsigargininduced vimentin expression in MDA-MB-468 breast cancer cells at concentrations of $3 \mu \mathrm{M}$ and above (Fig. 6a). Consistent with the inability of ORAI1 silencing to inhibit 
cytochalasin D-induced vimentin expression, YM58483 ( 3 or $10 \mu \mathrm{M}$ ) did not inhibit vimentin expression induced by cytochalasin D (Fig. 6b). Paradoxically, unlike ORAI1 and STIM1 siRNA treatments, YM58483 did not significantly reduce EGTA-AM-induced vimentin expression (Fig. 6c), perhaps suggesting an immediate compensatory role for
A
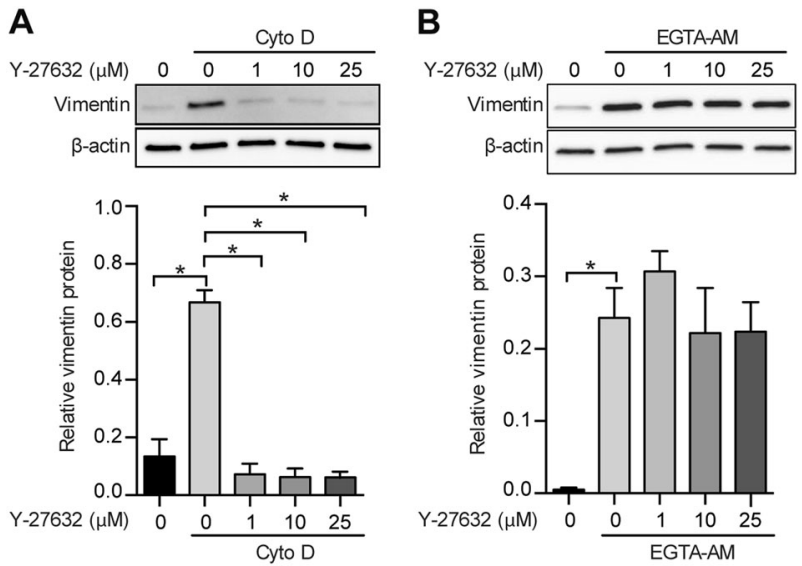

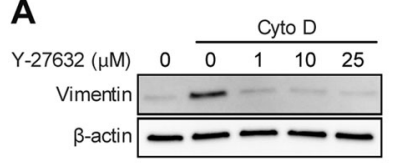

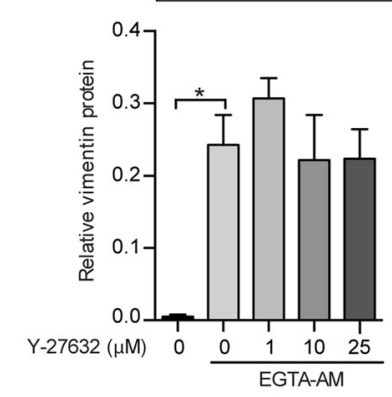

Fig. 2 Rho kinase/ROCK inhibition reduces cytochalasin D but not EGTA-AM induced vimentin expression. Representative immunoblot and densitometric analysis (normalized to $\beta$-actin) of the effect of increasing concentrations of the Rho kinase/ROCK inhibitor, Y-27632 $(0,1,10$, and $25 \mu \mathrm{M})$, on a cytochalasin D (cyto D, $300 \mathrm{nM}, 24 \mathrm{~h}$ ) and b EGTA-AM $(100 \mu \mathrm{M}, 1 \mathrm{~h})$ induced vimentin protein expression at $24 \mathrm{~h}$. Cells were preincubated with Y-27632 for $1 \mathrm{~h}$ prior to treatment with either EGTA-AM or cyto D, with Y-27632 maintained for the duration of the experiment. Bar graphs represent the mean $\pm \mathrm{SD}$ for three independent experiments. $P<0.05$ (one-way ANOVA with Tukey's multiple comparisons test)
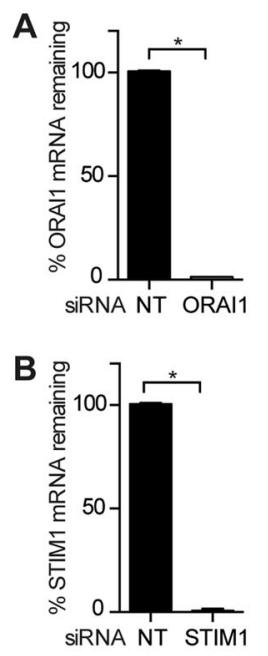

C
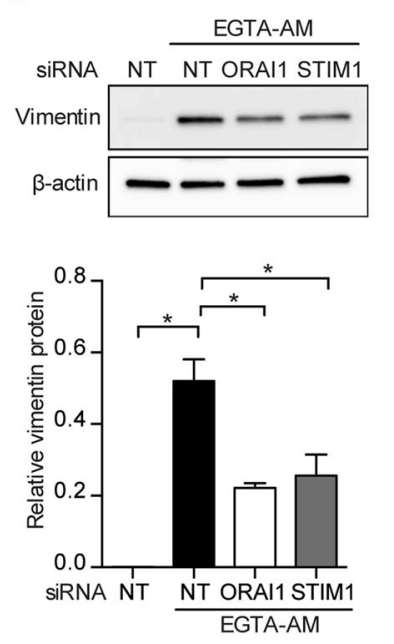

D
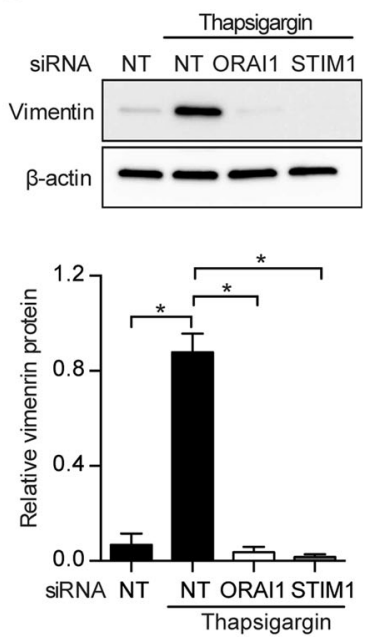

E
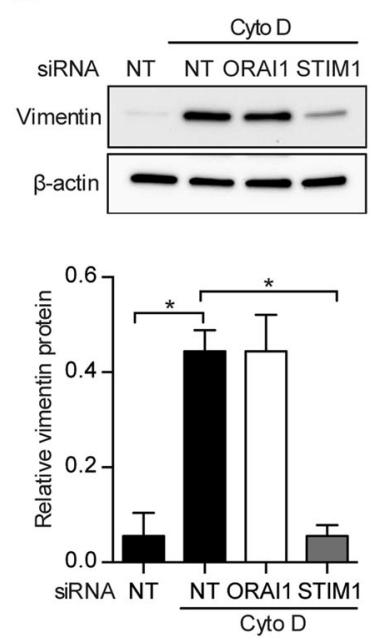

Fig. 3 The major store-operated calcium entry components ORAI1 and STIM1, differentially regulate vimentin protein expression in MDA-MB-468 breast cancer cells induced by EGTA-AM, cytochalasin D, and thapsigargin. Analysis of percent remaining a ORAI1 and b STIM1 mRNA in MDA-MB-468 cells following siRNA-mediated silencing. Bar graphs represent the mean \pm SD for three independent experiments. $P<0.05$ (unpaired $t$-test). Representative immunoblot

and densitometric analysis (normalized to $\beta$-actin) of the effect of ORAI1 and STIM1 silencing on c EGTA-AM $(100 \mu \mathrm{M}, 1 \mathrm{~h})$, d thapsigargin $(100 \mathrm{nM}, 24 \mathrm{~h})$, and e cytochalasin D (cyto D, $300 \mathrm{nM}$, $24 \mathrm{~h}$ ) induced vimentin protein expression in MDA-MB-468 breast cancer cells at $24 \mathrm{~h}$. Bar graphs represent the mean \pm SD for three independent experiments. $P<0.05$ (one-way ANOVA with Tukey's multiple comparisons test); NT nontargeting 

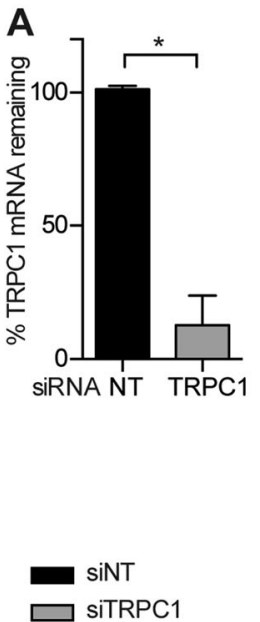

B

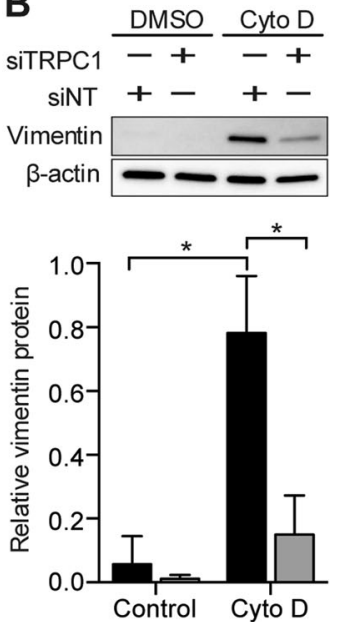

C
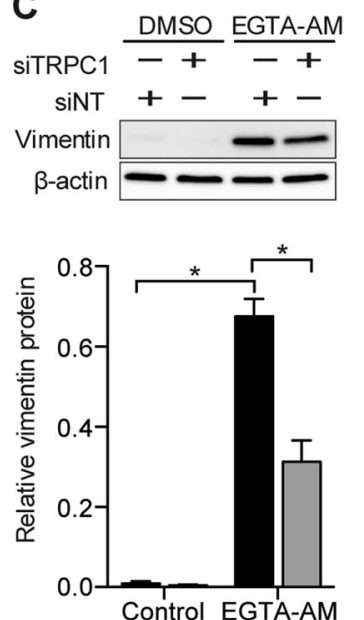

D
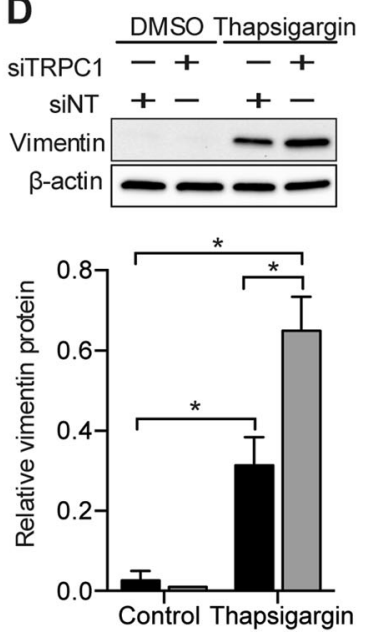

Fig. 4 TRPC1 silencing reduces cytochalasin D but not thapsigargininduced vimentin expression in MDA-MB-468 cells. a Analysis of percent remaining TRPC1 mRNA in MDA-MB-468 cells following siRNA-mediated silencing $(24 \mathrm{~h})$. Bar graphs represent the mean \pm SD for three independent experiments. $P<0.05$ (unpaired $t$-test). Representative immunoblot showing the effect of TRPC1 siRNA-mediated

silencing on b cytochalasin D (cyto D, $300 \mathrm{nM}, 24 \mathrm{~h}$ ), c EGTA-AM $(100 \mu \mathrm{M}, 1 \mathrm{~h})$, and $\mathbf{d}$ thapsigargin $(100 \mathrm{nM}, 24 \mathrm{~h})$ induced vimentin expression (normalized to $\beta$-actin) in MDA-MB-468 breast cancer cells at $24 \mathrm{~h}$. Bar graphs represent the mean \pm SD for three independent experiments. $P<0.05$ (one-way ANOVA with Tukey's multiple comparisons test); NT nontargeting

ion channels implicated in EMT induction in a variety of different cancer models warrants a deeper understanding of how different EMT inducers engage different $\mathrm{Ca}^{2+}$ influx pathways, and which of these are indispensable to EMT induction.

This study demonstrated the ability of the slow $\mathrm{Ca}^{2+}$ buffer EGTA-AM to induce vimentin expression via an ORAI1-dependent pathway in MDA-MB-468 breast cancer cells. This may be due to a reduction in endoplasmic reticulum $\mathrm{Ca}^{2+}$ levels, subsequent STIM1-mediated activation of ORAI1 and a local increase in $\mathrm{Ca}^{2+}$ at the plasma membrane near ORAI channels, which would not be buffered by EGTA-AM [26]. Such a mechanism is supported by the ability of STIM1 silencing to attenuate EGTA-AMinduced vimentin expression and the ability of the storeoperated $\mathrm{Ca}^{2+}$ entry inducer thapsigargin to increase vimentin levels. This phenomenon is somewhat analogous to the selective activation of the $\mathrm{Ca}^{2+}$ dependent transcription factor NFAT1 by ORAI1 through $\mathrm{Ca}^{2+}$ increases near open ORAI1 channels [39]. Hence, localized calcium increases induced by ORAI1 activation may also be important in the induction of vimentin expression.

Despite ORAI1 not being a regulator of EGF-induced vimentin expression in MDA-MB-468 breast cancer cells [5], ORAI1-mediated $\mathrm{Ca}^{2+}$ influx is sufficient to promote vimentin expression. ORAI1 should therefore now be assessed in the context of other EMT inducers in this and other models. Our studies have also provided further evidence for the engagement of different cellular signaling pathways to promote vimentin expression through our comparison of vimentin expression induced by EGTA-AM and cytochalasin D.

In contrast to EGTA-AM, cytochalasin D-induced vimentin expression was sensitive to ROCK inhibition but was completely insensitive to ORAI1 silencing. However, the ability of STIM1 silencing to also attenuate cytochalasin D-induced vimentin expression was indicative of an endoplasmic $\mathrm{Ca}^{2+}$ store-dependent component, since STIM1 is an endoplasmic reticulum $\mathrm{Ca}^{2+}$ sensor. Our observation that TRPC1 silencing reduced cytochalasin D-induced vimentin expression is consistent with studies in other cell types that have shown that this ion channel can be a partner in STIM1-mediated events [21-24]. However, in contrast to reports in human umbilical vein endothelial cells and other models [40], TRPC1 does not contribute to store-operated $\mathrm{Ca}^{2+}$ entry in MDA-MB468 breast cancer cells [34]. This lack of contribution of TRPC1 to store-operated $\mathrm{Ca}^{2+}$ entry in MDA-MB-468 breast cancer cells provides more evidence for the delineation between the signaling pathways induced by thapsigargin and cytochalasin $\mathrm{D}$ to promote vimentin expression in this model.

Our studies also provide evidence that some stimuli may engage both TRPC1- and ORAI1-dependent $\mathrm{Ca}^{2+}$ influx to induce vimentin expression. This is demonstrated by the ability of both ORAI1 and TRPC1 silencing to partially reduce vimentin expression induced by EGTA-AM. Further studies are required to determine exactly how cytochalasin D and thapsigargin achieve specific activation of TRPC1 and ORAI1, respectively, in this model of breast cancer plasticity. However, possible mechanisms are (i) the dramatic cytoskeletal and morphological remodeling induced by cytochalasin D drives different spatial localization of STIM1, which promotes distinct engagement with ORAI1 and TRPC1, and/or 
A

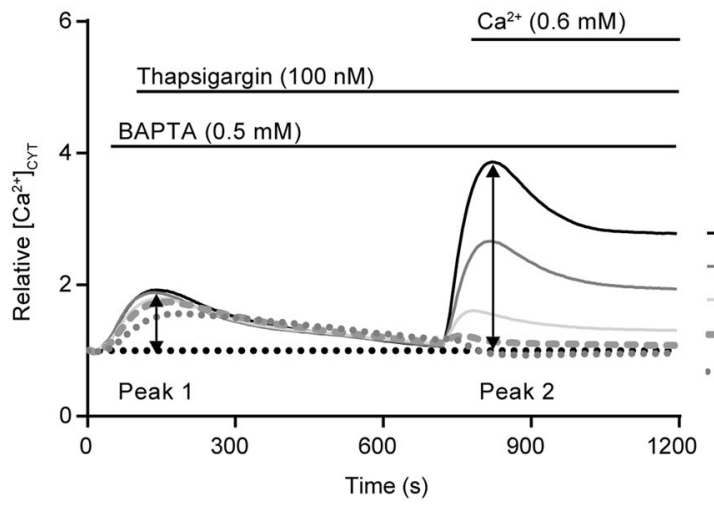

Fig. 5 YM58483-mediated pharmacological inhibition of storeoperated calcium entry in MDA-MB-468 cells. a Average relative $\left[\mathrm{Ca}^{2+}\right]_{\mathrm{CYT}}$ transients and $\mathbf{b}$ ratio of Peak 2/Peak 1 (maximum peak height, as indicated in panel (A)) in MDA-MB-468 cells pretreated $(15 \mathrm{~min})$ with increasing concentrations of the store-operated $\mathrm{Ca}^{2+}$
B

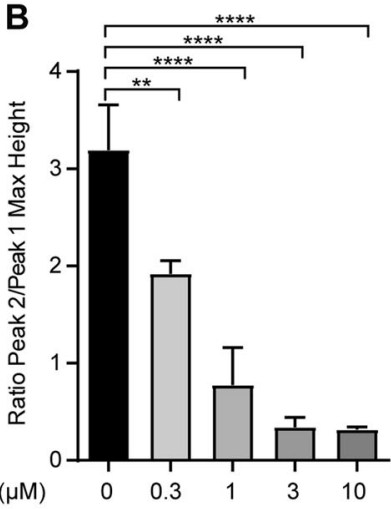

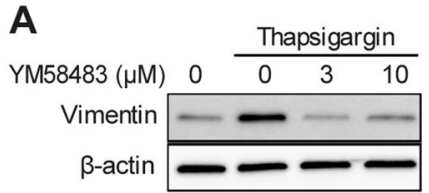

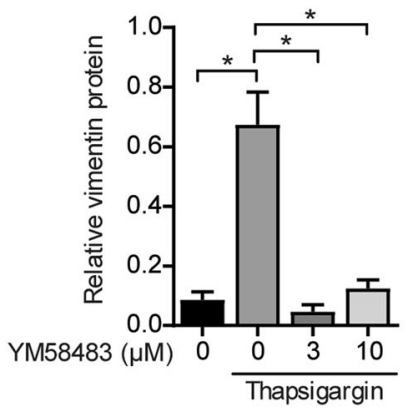

Fig. 6 The selective store-operated calcium influx inhibitor YM58483 completely blocks thapsigargin but not EGTA-AM induced vimentin expression in MDA-MB-468 cells. Representative immunoblot and densitometric analysis of the effect of the store-operated $\mathrm{Ca}^{2+}$ influx inhibitor, YM58483, on a thapsigargin $(100 \mathrm{nM})$, b cytochalasin D (cyto D, $300 \mathrm{nM})$, and c EGTA-AM $(100 \mu \mathrm{M}, 1 \mathrm{~h})$ induced vimentin entry inhibitor, YM58483. Inhibitor concentrations were maintained for the duration of the experiment (1200 s). Bar graphs represent the mean $\pm \mathrm{SD}$ for three independent experiments. $P<0.05$ (one-way ANOVA with Tukey's multiple comparisons test)
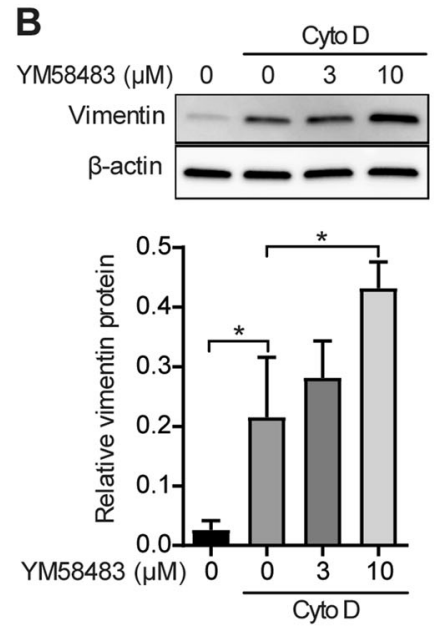
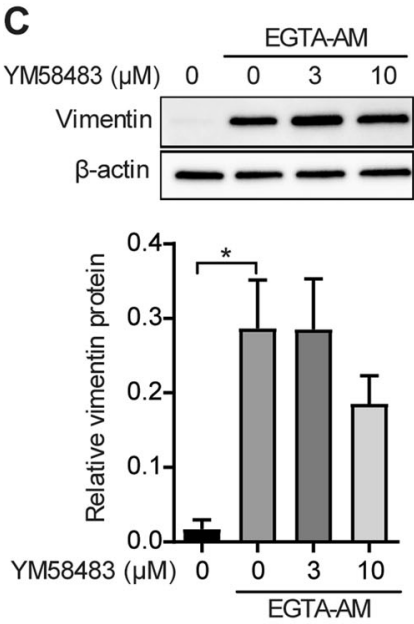

protein expression $(24 \mathrm{~h})$. MDA-MB-468 cells were pretreated for $1 \mathrm{~h}$ with inhibitor prior to treatment with either thapsigargin, cyto $\mathrm{D}$, or EGTA-AM and inhibitor was maintained for the duration of the experiment. Bar graphs represent the mean \pm SD for three independent experiments. $P<0.05$ (one-way ANOVA with Tukey's multiple comparisons test)

(ii) cytochalasin $\mathrm{D}$ induces other signaling pathways that drive a more TRPC1-dependent pathway. The inability of selective pharmacological inhibition of store-operated $\mathrm{Ca}^{2+}$ entry to inhibit cytochalasin D-induced vimentin expression was expected since this mechanism of vimentin expression induction was also insensitive to ORAI1 silencing. Despite the ability of ORAI1 silencing to also attenuate EGTA-AMinduced vimentin expression, this induction was insensitive to pharmacological inhibition of store-operated $\mathrm{Ca}^{2+}$ entry at concentrations of YM58483 that almost abolished thapsigargin-induced vimentin expression. The inability of YM58483 to inhibit EGTA-AM-induced vimentin expression may be due to TRPC1-dependent $\mathrm{Ca}^{2+}$ influx that may have compensated for the short-term pharmacological inhibition of ORAI1. Alternatively, it may be further evidence of a pathway involving STIM1-ORAI1 interactions that are independent of $\mathrm{Ca}^{2+}$ entry via the store-operated $\mathrm{Ca}^{2+}$ entry mechanism. Indeed, noncanonical activation of ORAI channels has been reported in other models [41-43].

Although ORAI1 and TRPC1 are clearly associated with vimentin induction by thapsigargin and cytochalasin $\mathrm{D}$, respectively, in MDA-MB-468 breast cancer cells, these $\mathrm{Ca}^{2+}$ influx pathways may not regulate all EMT markers to the same extent. Indeed, the $\mathrm{Ca}^{2+}$ permeable ion channel TRPM7 regulates vimentin protein expression induced by EGF but not another EMT marker, Twist, in MDA-MB-468 breast cancer 


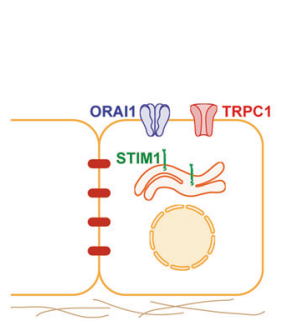

Epithelial Cell

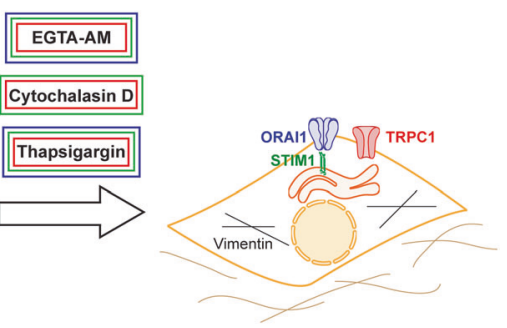

Mesenchymal Cell

Fig. 7 Schematic representation of $\mathrm{Ca}^{2+}$-dependent pathways via which EGTA-AM, cytochalasin D, and thapsigargin may act to induce vimentin expression in MDA-MB-468 cells. Blue box, ORAI1dependent pathway; green box, STIM1-dependent pathway; red, TRPC1-dependent pathway

Table 1 Summary of the effect of siRNA-mediated silencing of storeoperated $\mathrm{Ca}^{2+}$ entry components on EGTA-AM, cytochalasin D (cyto D), and thapsigargin-induced vimentin expression in MDA-MB468 cells

\begin{tabular}{llll}
\hline & siORAI1 & siSTIM1 & siTRPC1 \\
\hline EGTA-AM & $\downarrow$ & $\downarrow$ & $\downarrow$ \\
Cyto D & $\leftrightarrow$ & $\downarrow$ & $\downarrow$ \\
Thapsigargin & $\downarrow$ & $\downarrow$ & $\uparrow$ \\
\hline
\end{tabular}

Thapsigargin and cytochalasin D both use a STIM1-dependent pathway for vimentin expression in MDA-MB-468 breast cancer cells but differentially engage ORAI1 and TRPC1. EGTA-AM-induced vimentin expression is STIM1, ORAI1, and TRPC1 sensitive. $\uparrow$, increased; $\downarrow$, decreased; $\leftrightarrow$ no change, vimentin expression relative to non-targeting control

cells [5]. The nature of the stimuli therefore also determines which EMT markers are induced. In the complex in vivo tumor microenvironment, cancer cells are likely to simultaneously receive and process multiple microenvironmental cues (e.g., growth factor signaling, oxygen stress, and mechanical cues), each of which may engage different signal transduction pathways to orchestrate a larger EMT program. Another major contributing factor may be the cell type. Cytochalasin D alone was not an inducer of vimentin expression in other EMT-sensitive cell lines that we examined (PMC42LA and A431, data not shown). This suggests that cytochalasin D induces EMT only when there is a specific interplay between the expression and localization of specific ion channels and cytochalasin D-induced changes in cell morphology and/or signaling. Hence, future studies should assess other models of EMT to define what features are important in this phenomenon. The heterogeneity in vimentin expression induction that we identified with different stimuli is summarized in Fig. 7 and Table 1. This heterogeneity may represent a significant challenge in the therapeutic targeting of $\mathrm{Ca}^{2+}$ permeable ion channels for the control of metastatic progression and/or therapeutic resistance via EMT, if tumor microenvironmental factors also engage different $\mathrm{Ca}^{2+}$ permeable ion channels. Future studies should now focus on

the identification of $\mathrm{Ca}^{2+}$ permeable ion channels for which pharmacological inhibition can individually or cooperatively suppress specific features of EMT across multiple stimuli and breast cancer models.

Acknowledgements We thank Dr Felicity Davis for her helpful comments and assistance in proofreading this manuscript. This work was supported by the National Health and Medical Research Council (NHMRC) of Australia (1022263, 1079672). GRM was supported by the Mater Foundation. TAS was funded by an NHMRC Biomedical Postgraduate Scholarship (1039358). EWT was supported in part by a grant from the National Breast Cancer Foundation. The Translational Research Institute is supported by a grant from the Australian Government.

\section{Compliance with ethical standards}

Conflict of interest GRM and SR-T have patents related to ORAI1 in breast cancer therapy.

Publisher's note: Springer Nature remains neutral with regard to jurisdictional claims in published maps and institutional affiliations.

\section{References}

1. Ye X, Weinberg RA. Epithelial-mesenchymal plasticity: a central regulator of cancer progression. Trends Cell Biol. 2015;25:675-86.

2. Hollier BG, Evans K, Mani SA. The epithelial-to-mesenchymal transition and cancer stem cells: a coalition against cancer therapies. J Mammary Gland Biol Neoplasia. 2009;14:29-43.

3. Bonnomet A, Syne L, Brysse A, Feyereisen E, Thompson EW, Noel A, et al. A dynamic in vivo model of epithelial-tomesenchymal transitions in circulating tumor cells and metastases of breast cancer. Oncogene 2012;31:3741-53.

4. Quail DF, Joyce JA. Microenvironmental regulation of tumor progression and metastasis. Nat Med. 2013;19:1423-37.

5. Davis FM, Azimi I, Faville RA, Peters AA, Jalink K, Putney JW Jr., et al. Induction of epithelial-mesenchymal transition (EMT) in breast cancer cells is calcium signal dependent. Oncogene 2014;33:2307-16.

6. Morel AP, Hinkal GW, Thomas C, Fauvet F, Courtois-Cox S, Wierinckx A, et al. EMT inducers catalyze malignant transformation of mammary epithelial cells and drive tumorigenesis towards claudin-low tumors in transgenic mice. PLoS Genet. 2012;8:e1002723.

7. Mani SA, Guo W, Liao MJ, Eaton EN, Ayyanan A, Zhou AY, et al. The epithelial-mesenchymal transition generates cells with properties of stem cells. Cell 2008;133:704-15.

8. May CD, Sphyris N, Evans KW, Werden SJ, Guo W, Mani SA. Epithelial-mesenchymal transition and cancer stem cells: a dangerously dynamic duo in breast cancer progression. Breast Cancer Res. 2011;13:202.

9. Ivaska J, Pallari HM, Nevo J, Eriksson JE. Novel functions of vimentin in cell adhesion, migration, and signaling. Exp Cell Res. 2007;313:2050-62.

10. Davis FM, Stewart TA, Thompson EW, Monteith GR. Targeting EMT in cancer: opportunities for pharmacological intervention. Trends Pharmacol Sci. 2014;35:479-88.

11. Luo M, Brooks M, Wicha MS. Epithelial-mesenchymal plasticity of breast cancer stem cells: implications for metastasis and therapeutic resistance. Curr Pharm Des. 2015;21:1301-10.

12. Davis FM, Kenny PA, Soo ET, van Denderen BJ, Thompson EW, Cabot PJ, et al. Remodeling of purinergic receptor-mediated $\mathrm{Ca}^{2+}$ 
signaling as a consequence of EGF-induced epithelial-mesenchymal transition in breast cancer cells. PLoS ONE. 2011;6:e23464.

13. Liu J, Chen Y, Shuai S, Ding D, Li R, Luo R. TRPM8 promotes aggressiveness of breast cancer cells by regulating EMT via activating AKT/GSK-3beta pathway. Tumour Biol. 2014;35:8969-77.

14. Wen L, Liang C, Chen E, Chen W, Liang F, Zhi X, et al. Regulation of multi-drug Resistance in hepatocellular carcinoma cells is TRPC6/calcium dependent. Sci Rep. 2016;6:23269.

15. Azimi I, Monteith GR. Plasma membrane ion channels and epithelial to mesenchymal transition in cancer cells. Endocr Relat Cancer. 2016;23:R517-25.

16. Zhu S, Zhou HY, Deng SC, Deng SJ, He C, Li X, et al. ASIC1 and ASIC3 contribute to acidity-induced EMT of pancreatic cancer through activating $\mathrm{Ca}(2+) / \mathrm{RhoA}$ pathway. Cell Death Dis. 2017;8:e2806.

17. Wu Y, Xu X, Ma L, Yi Q, Sun W, Tang L. Calreticulin regulates TGF-beta1-induced epithelial mesenchymal transition through modulating Smad signaling and calcium signaling. Int J Biochem Cell Biol. 2017;90:103-13.

18. Qi L, Song W, Li L, Cao L, Yu Y, Song C, et al. FGF4 induces epithelial-mesenchymal transition by inducing store-operated calcium entry in lung adenocarcinoma. Oncotarget. 2016;7:74015-30.

19. Cai X, Zhou Y, Nwokonko RM, Loktionova NA, Wang X, Xin P, et al. The Orail store-operated calcium channel functions as a hexamer. J Biol Chem. 2016;291:25764-75.

20. Putney JW, Steinckwich-Besancon N, Numaga-Tomita T, Davis FM, Desai PN, D'Agostin DM, et al. The functions of storeoperated calcium channels. Biochim Biophys Acta Mol Cell Res. 2017;1864:900-6.

21. Ambudkar IS, de Souza LB, Ong HL. TRPC1, Orai1, and STIM1 in SOCE: friends in tight spaces. Cell Calcium. 2017;63:33-9.

22. Ng LC, O'Neill KG, French D, Airey JA, Singer CA, Tian H, et al. TRPC1 and Orai1 interact with STIM1 and mediate capacitative $\mathrm{Ca}(2+)$ entry caused by acute hypoxia in mouse pulmonary arterial smooth muscle cells. Am J Physiol Cell Physiol. 2012;303:C1156-72.

23. Pani B, Ong HL, Brazer SC, Liu X, Rauser K, Singh BB, et al. Activation of TRPC1 by STIM1 in ER-PM microdomains involves release of the channel from its scaffold caveolin-1. Proc Natl Acad Sci USA. 2009;106:20087-92.

24. Shi J, Miralles F, Birnbaumer L, Large WA, Albert AP. Storeoperated interactions between plasmalemmal STIM1 and TRPC1 proteins stimulate PLCbeta1 to induce TRPC1 channel activation in vascular smooth muscle cells. J Physiol. 2017;595:1039-58.

25. Azimi I, Milevskiy MJG, Kaemmerer E, Turner D, Yapa K, Brown MA, et al. TRPC1 is a differential regulator of hypoxiamediated events and Akt signalling in PTEN-deficient breast cancer cells. J Cell Sci. 2017;130:2292-305.

26. Naraghi $\mathrm{M}$, Neher E. Linearized buffered $\mathrm{Ca}^{2+}$ diffusion in microdomains and its implications for calculation of $\left[\mathrm{Ca}^{2+}\right]$ at the mouth of a calcium channel. J Neurosci. 1997;17:6961-73.

27. Metzen E, Fandrey J, Jelkmann W. Evidence against a major role for $\mathrm{Ca}^{2+}$ in hypoxia-induced gene expression in human hepatoma cells (Hep3B). J Physiol. 1999;517(Pt 3):651-7.

28. Waser M, Mesaeli N, Spencer C, Michalak M. Regulation of calreticulin gene expression by calcium. J Cell Biol. 1997;138:547-57.
29. Ouanounou A, Zhang L, Charlton MP, Carlen PL. Differential modulation of synaptic transmission by calcium chelators in young and aged hippocampal CA1 neurons: evidence for altered calcium homeostasis in aging. J Neurosci. 1999;19:906-15.

30. Ouanounou A, Zhang L, Tymianski M, Charlton MP, Wallace MC, Carlen PL. Accumulation and extrusion of permeant $\mathrm{Ca}^{2+}$ chelators in attenuation of synaptic transmission at hippocampal CA1 neurons. Neuroscience. 1996;75: 99-109.

31. Aung CS, Ye W, Plowman G, Peters AA, Monteith GR, RobertsThomson SJ. Plasma membrane calcium ATPase 4 and the remodeling of calcium homeostasis in human colon cancer cells. Carcinogenesis. 2009;30:1962-9.

32. Gallo RM, Khan MA, Shi J, Kapur R, Wei L, Bailey JC, et al. Regulation of the actin cytoskeleton by Rho kinase controls antigen presentation by CD1d. J Immunol. 2012;189:1689-98.

33. Liao JK, Seto M, Noma K. Rho kinase (ROCK) inhibitors. J Cardiovasc Pharmacol. 2007;50:17-24.

34. Davis FM, Peters AA, Grice DM, Cabot PJ, Parat MO, RobertsThomson SJ, et al. Non-stimulated, agonist-stimulated and storeoperated $\mathrm{Ca}^{2+}$ influx in MDA-MB-468 breast cancer cells and the effect of EGF-induced EMT on calcium entry. PLoS ONE. 2012;7:e36923.

35. Azimi I, Bong AH, Poo GXH, Armitage K, Lok D, RobertsThomson SJ, et al. Pharmacological inhibition of store-operated calcium entry in MDA-MB-468 basal A breast cancer cells: consequences on calcium signalling, cell migration and proliferation. Cell Mol Life Sci. 2018;75:4525-37.

36. Azimi I, Flanagan JU, Stevenson RJ, Inserra M, Vetter I, Monteith GR, et al. Evaluation of known and novel inhibitors of Orai1mediated store operated $\mathrm{Ca}(2+)$ entry in MDA-MB-231 breast cancer cells using a Fluorescence Imaging Plate Reader assay. Bioorg Med Chem. 2017;25:440-9.

37. Bhatia S, Monkman J, Toh AKL, Nagaraj SH, Thompson EW. Targeting epithelial-mesenchymal plasticity in cancer: clinical and preclinical advances in therapy and monitoring. Biochem $\mathrm{J}$. 2017;474:3269-306.

38. Redfern AD, Spalding LJ, Thompson EW. The Kraken Wakes: induced EMT as a driver of tumour aggression and poor outcome. Clin Exp Metast. 2018;35:285-308.

39. Kar P, Parekh AB. Distinct spatial $\mathrm{Ca}^{2+}$ signatures selectively activate different NFAT transcription factor isoforms. Mol Cell. 2015;58:232-43.

40. Cheng KT, Liu X, Ong HL, Ambudkar IS. Functional requirement for Orai1 in store-operated TRPC1-STIM1 channels. J Biol Chem. 2008;283:12935-40.

41. Dubois C, Vanden Abeele F, Lehen'kyi V, Gkika D, Guarmit B, Lepage $\mathrm{G}$, et al. Remodeling of channel-forming ORAI proteins determines an oncogenic switch in prostate cancer. Cancer Cell. 2014;26:19-32.

42. Feng M, Grice DM, Faddy HM, Nguyen N, Leitch S, Wang Y, et al. Store-independent activation of Orai1 by SPCA2 in mammary tumors. Cell 2010;143:84-98.

43. Latour S, Mahouche I, Cherrier F, Azzi-Martin L, Velasco V, Soubeyran P, et al. Calcium independent effect of Orail and STIM1 in non-Hodgkin B cell lymphoma dissemination. Cancers 2018;10:E402. 